\title{
Malignant change in an intraspinal nerve sheath tumour
}

\author{
D. J. B. ASHLEY AND P. J. E. WILSON \\ From the Departments of Pathology and Neurosurgery, Morriston Hospital, Swansea
}

SUMMARY A spinal neurofibroma with malignant change leading to 'sarcomatous meningitis' is described in a woman of 73. Three similar cases have been found in the literature. This is an exceedingly rare complication of nerve sheath tumours in the spinal canal.

Primary intraspinal tumours are less common than primary intracranial neoplasms and are more often derived from extra-medullary tissues. Kernohan and Sayre (1952) described a collection of 979 intraspinal lesions, more than half of which were nerve sheath tumours (293 cases) and meningiomas ( 254 cases). Russell and Rubinstein (1963) likewise found nerve sheath tumours to be as frequent as meningiomas in the spinal canal. Hosoi (1931) found that $13 \%$ of patients with neurofibromatosis eventually developed neurofibrosarcomata and Stout (1949) found that over half of the neurofibrosarcomata reported had arisen in nodules of neurofibromatosis. Malignant change in solitary or ostensibly solitary nerve sheath tumours is rare. Having been able to find only three such cases in the literature, we feel justified in reporting a further case because of the rarity of the condition and because of its misleading clinical presentation.

\section{CASE HISTORY}

A widow aged 73 years was referred to one of us (P.J.E.W.) on 19 December 1968 because of severe weakness and numbness of both legs, of one day's duration.

She had been an alert and energetic woman until November 1968, when she began to suffer from bouts of severe frontal headache accompanied by nausea and vomiting. Her relatives then noticed that she was mentally confused, rambling, and at times drowsy. On admission to hospital on 6 December 1969, she was found to be a well-nourished, alert woman, but disorientated and unable to give a clear account of herself. Her initial alertness gave place to fluctuating drowsiness. Neurological examination showed no papilloedema, no defects of cranial nerve functions, no weakness, tremor, dystonia, or incoordination of the limbs, no abnormality of limb reflexes, and no overt sensory disturbance, but the left plantar response was extensor. General physical examination was not contributory; there was no evidence of generalized neurofibromatosis. The peripheral blood was normal, the ESR $18 \mathrm{~mm}$ in one hour, and serological tests were negative. Skull radiographs showed no abnormality. The chest radiograph showed slight unfolding of the aorta and a small indefinite opacity in the lower zone of the right lung-field. Lumbar puncture revealed yellow CSF at lowo pressure, containing protein $3300 \mathrm{mg} / 100 \mathrm{ml}$., no redo cells, and 1 white cell per c.mm. No manometric block was found on jugular compression.

The patient was seen by Dr. B. M. Phillips, consultan neurologist, who suspected the presence of a chronico subdural haematoma. However, an ultrasonic echogram showed no shift of the midline echo, and an EEG showed no lateralized abnormality, although it was grossly abnormal by virtue of persistent high-amplitude delta activity at 1 to 2 .Hz over both fronto-temporal regions. Left carotid angiography showed irregularity and tortuosity of the carotid syphon and its major branches, particularly the pericallosal artery. Right carotid angiography failed.

On 18 December 1968 the patient was noticed for the first time to have difficulty in walking. By the following day, she exhibited a flaccid paraparesis, more dense in the right leg than the left, with bilateral extensor plantar responses, a sensory level to pinprick at T6 dermatome and acute urinary retention. Myelography revealed a complete spinal block opposite $\mathrm{T} 5$ vertebral body, with a 'smear' effect for some four segments caudal to this, suggestive of arachnoidal adhesions (Fig. 1).

Immediate spinal exploration was performed by one of us (P.J.E.W.), the laminae of T2 to T6 vertebrae inclusive being removed. No extradural abnormality was found, but opening the dura at the level of T4 to 5 revealed a large sausage-shaped, firm, pink, smooth tumour, displacing the spinal cord forwards and to the left. The pia-arachnoid was thickened and infiltrated in a nodular fashion by pink and yellow tissue almost to the limits of the operative exposure, with here and there small loculi of clear yellow fluid. The tumour was easily mobilized and removed in toto, together with posterior rootlets of the 


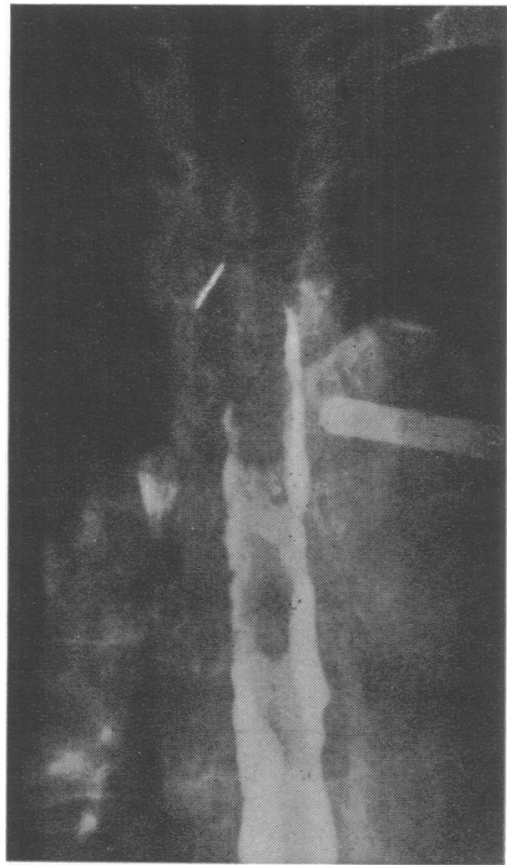

FIG. 1. Myelogram showing a complete spinal block at the level of the 5th thoracic vertebra.

right T5 spinal root from which it clearly arose. As much as possible of the infiltrated arachnoid was also removed, which amply decompressed the cord but did not restore cerebrospinal fluid flow.

For the first week after operation there was both motor and sensory improvement in the lower limbs, and she was more continuously alert, lucid, and better orientated. Thereafter an insidious deterioration occurred, until by 6 January 1969 she was more densely paraplegic and/ more severely confused than she had been before laminectomy. Repeated myelography showed a total spinal block, below the limit of the laminectomy.

At this point it was conjectured that there was leptomeningeal seeding of neoplastic cells not only in the spinal canal but also above the foramen magnum, and that she might have a resultant communicating hydrocephalus which was responsible for the cerebral component of her illness. Diversion of the CSF and radiotherapy were therefore considered, but finally rejected in view of her generally poor condition and the reluctance of her family that she should undergo further surgery. She died suddenly on 11 January 1969.

HISTOLOGY The surgical specimen consisted of two parts. One was an ovoid smooth firm pink tumour measuring $2 \times 1 \times 1 \mathrm{~cm}$. The remainder comprised fragments of thickened nodular pia-arachnoid.

The solid tumour presented the features of a neurofibroma. It consisted for the most part of spindle-shaped cells arranged in interlacing bundles in a collagenous matrix (Fig. 2). In some areas there were groups of polygonal cells with foamy cytoplasm. Mitoses were infrequent and the cellular pattern was regular. There was a thin capsule. In several areas close to the capsule the histological appearance was different. Here the tumour consisted of polygonal cells with nuclei of irregular shapes and sizes (Fig. 3). The stroma comprised some collagen

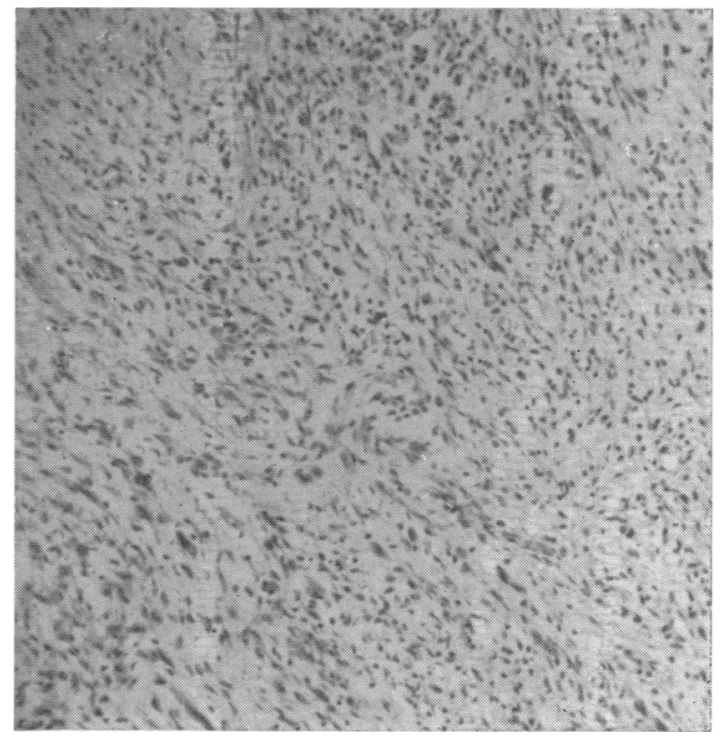

FIG. 2. A general view of the spinal tumour showing spindle shaped cells and groups of polygonal xanthomatous cells. $H$ and $E \times 100$.

fibres and masses of intercellular connective tissue mucin.

The fragment from the pia-arachnoid consisted of meningeal tissue which was heavily infiltrated with neoplastic cells resembling those in the poorly differentiated part of the main tumour.

The histological diagnosis made was neurofibroma of an intraspinal nerve root with malignant change and extension to the pia-arachnoid.

POST-MORTEM FINDINGS Permission for necropsy was confined to examination of the central nervous system.

The spinal canal was opened and the cord, with its meningeal investment, was removed. There was a depression in the posterolateral surface of the cord at the site of the original operation and a further small nodule $5 \times 3 \times 2 \mathrm{~mm}$ in size was found two segments below the depression. The pia and arachnoid membranes over the whole of the spinal cord were thickened and formed a white-pink layer about $1 \mathrm{~mm}$ thick.

The brain appeared, on macroscopic examination, to be normal, apart from a faint grey cloudiness of the meninges over the cerebellum. The cerebral ventricles were of normal size. 


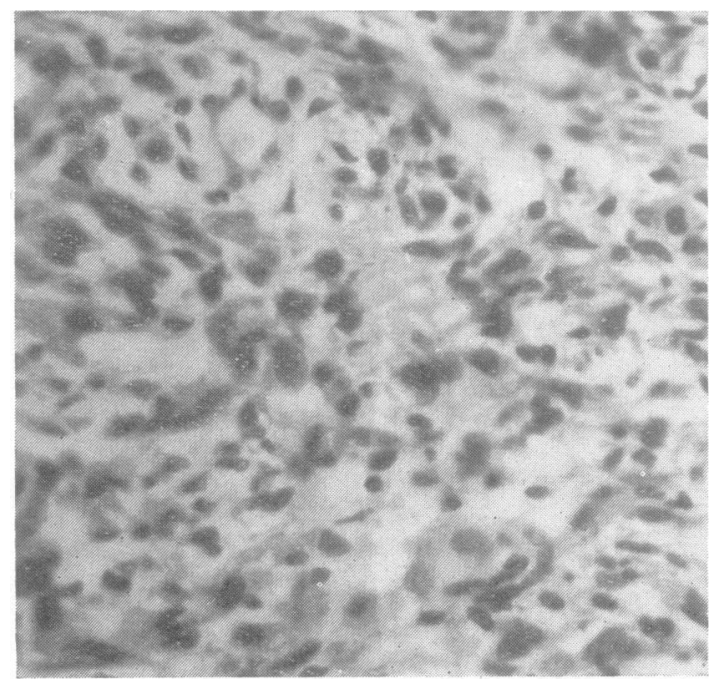

FIG. 3. Detail of the sarcomatous part of the primary tumour showing cellular irregularity and a lack of pattern. $H$ and $E, \times 400$.

Histological examination showed the small firm nodule in the meninges to have the same histological features as the tumour removed at operation, except that it merged into the neoplastic infiltration of the meninges. The meninges covering the whole cord showed infiltration with polygonal neoplastic cells with irregular hyperchromatic nuclei. This malignant tissue surrounded the nerve roots and vessels of the meninges but did not invade the substance of the spinal cord.

Sections of the brain showed a similar neoplastic invasion of the meninges of the cerebellum (Fig. 4). The extent of this infiltration was very much less than around the spinal cord and in places was only two or three cells thick. No invasion of the cerebellum was seen. No tumour was seen in the meninges over the cerebrum.

\section{DISCUSSION}

This patient's presentation with headache, vomiting, fluctuating mental changes, and drowsiness for about a month led to investigation for primary intracranial disease. The symptoms referable to a spinal lesion appeared abruptly and unexpectedly late in the disease and progressed with unusual rapidity. An early clue to the possibility of a spinal lesion was given by the very high level of protein in the CSF but this was misconstrued because of the absence of spinal symptoms and because manometry failed to show a spinal block.

The operative and necropsy findings showed that the primary disease was indeed spinal and tha malignant change had occurred in two separate nervo sheath tumours in the dorsal spine.

Two cases have been reported previously from Zürich (Brussatis and Zander, 1952; Zandef Barontini, and Brussatis, 1956). Both were women i⿱⺈

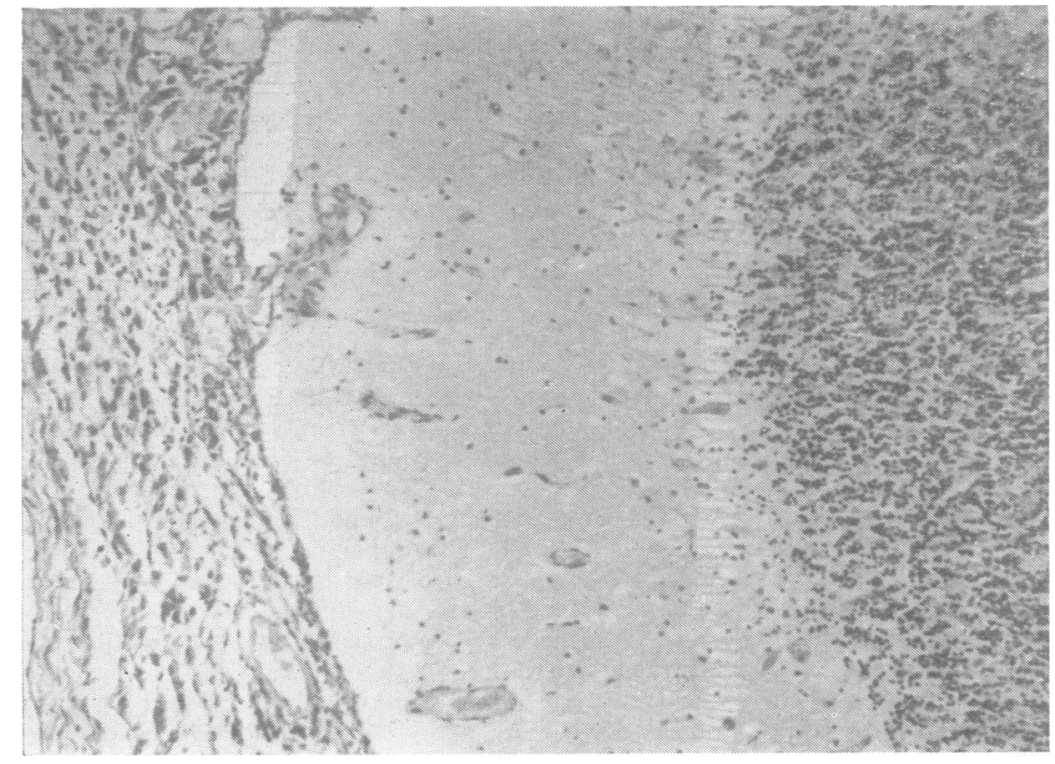

FIG. 4. The cerebellar cortex showing infiltration of the meninges by sarcomatous tissue. $H$ and $E, \times 100$. 
their late 30s and both gave a long history of pain in the back with the acute development of weakness and sensory loss in the lower limbs. In each case a laminectomy showed an encapsulated tumour in the lumbar spine, the lesion was removed and was found histologically to have the appearance of a benign Schwannoma. Recurrence occurred in each case, after eight and two years respectively, and reoperation showed tumours compressing the spinal cord. These tumours were histologically less well differentiated than the initial lesions and were diagnosed as neurofibrosarcomata. A further case fromZürich, reported at the same time, presented in a similar manner with a mass in the lower dorsal region which protruded through an intervertebral foramen into the mediastinum but this patient also showed generalized neurofibromatosis and therefore differs significantly from our patient.

The third case was recorded from Australia (Fowler, 1955); the patient was a man of 60 who gave six months' history of symptoms suggestive of spinal cord tumour and at operation a tumour was found filling the intrathecal space at the level of the cauda equina. Histologically this lesion was interpreted as a malignant neurilemmoma, there were areas which microscopically were typical of benign neurilemmoma and other areas in which there was a sharp transition to a less well-differentiated malignant form. This man died two weeks after the operation with some cerebral symptoms suggestive of raised intracranial pressure and papilloedema was noted. No necropsy was performed.

The present case differs somewhat from those previously reported as there was only a very short history of symptoms and surgical intervention was prompt. On histological examination there was very clear evidence that the lesion comprised malignant change in a preceding benign tumour and this was supported by the anatomical finding of discrete tumours which had indented the spinal cord. A striking feature was the spread of the tumour in the spinal meninges and its extension, presumably by the cerebrospinal fluid, to the meninges at the base of the brain. It is unfortunate that no necropsy was carried out in Fowler's case, as the cranial neurological signs suggest that a similar phenomenon may have occurred in this patient.

In retrospect, it is difficult to see any way in which the outcome in our patient could have been improved. The history was extremely short and the lesion was already beyond the scope of curative surgery at the time of diagnosis. This and the previously reported cases do, however, remind us that recurrence and malignant change, although rare, can occur in nerve sheath tumours of the spinal cord, that multiple asymptomatic tumours may coexist, and that these should be sought and, where surgically feasible, removed to avoid this complication. Dissemination of tumour cells through the CSF and the development of 'sarcomatous meningitis' was an interesting feature in our patient and was possibly the cause of the cerebral symptoms in the case reported by Fowler.

\section{REFERENCES}

Brusattis, F., and Zander, E. (1952). Über maligne Entartung spinaler Neurinome. Schweiz. Arch. Neurol. Psychiat., 70, 176-8.

Fowler, M. (1955). A malignant neurilemmoma. Med. J. Aust., 1, 236-7.

Hosoi, K. (1931). Multiple neurofibromatosis. Arch. Surg., 22, 258-281.

Kernohan, J. W., and Sayre, G. P. (1952). Tumors of the central nervous system. Section 10, Fascicle 35. Atlas of Tumor Pathology. Armed Forces Institute of Pathology: Washington, D.C.

Russell, D. S., and Rubinstein, L. J. (1963). Pathology of Tumours of the Nervous System, 2nd edn. Arnold: London. Stout, A.P. (1949). Tumours of the peripheral nervous system, Section 2, Fascicle 6. Atlas of Tumor Pathology. Armed Forces Institute of Pathology. Washington, D.C.

Zander, E., Barontini, F., and Brusattis, F. (1956). Sul problema della malignità nei neurinomi. Riv. Pat. nerv. ment., 77, 323-344. 